\title{
DONNE SVENTRATE E UOMINI SCUOIATI. CORPO FEMMINILE E CORPO MASCHILE NELLE CERE ANATOMICHE
}

\author{
DISEMBOWELLED WOMEN AND SKINNED MEN. FEMALE BODY AND MALE \\ BODY IN ANATOMICAL WAX FIGURES
}

Francesco Paolo De Ceglia

Universidad de Bari, Italia

\section{Riassunto:}

Nel 1775, viene inaugurata una mostra di "pezzi anatomici" ossia rappresentazioni plastiche di organi umani, al Museo della Specola di Firenze. La scultura senza dubbio più interessante della raccolta è la "Venere de' Medici". Tutti coloro che ammiravano questa opera, né rimanevano sconvolti. La vista di queste cere, è un'iniziazione agli uomini verso il mondo degli adulti. Per quanto riguarda gli "uomini scuoiati" viene rappresentato un "Adamo anatomico". Le differenze sono evidenti: la donna è passionale, personifica la morbidezza e la sua posizione è supina; l'uomo è atarassico (non mostra sofferenza), la sua posizione è eretta e rimanda alla durezza e alla verticalità.

\section{Palabras Claves:}

"pezzi anatomici", convenzione borghese, passionale, morbidezza, supina, atarassico, durezza, verticalità.

\section{Abstract:}

In 1775, an exhibition of "anatomical pieces" begins, namely plastic representations of human organs at the Specola Florence Museum. The most attractive sculpture is, with no doubt, the "Venus de' Medici". Everybody who admired this work remained totally amazed. The view of these waxes, leads men to the adult world. As regards "skinned men" (uomini scuoiati), it is represented an "Anatomical Adam" (Adamo anatomico). Differences are obvious: the woman is passionate, soft and her position is supine; the man does not show his pain and his position is upright. It refers to hardness and verticality.

\section{KEY WORD:}

"anatomical pieces", bourgeois convention, passionate, soft, supine, hardness, verticality. 


\section{Donne SVentrate}

La raccolta ceroplastica del Museo della Specola a Firenzeè ampia e variegata: nel 1775, anno di apertura, gli oggetti erano già 368; ora sono più di 2000. Accanto alle grandi statue a figura intera, cui si presterà attenzione, vi sono infatti numerosi "pezzi" anatomici, ossia rappresentazioni plastiche di singoli organi, apparati o regioni del corpo, che costituiscono la gran parte degli oggetti della raccolta. Nonostante la loro abbondanza, essi fungono tuttavia solo da sfondo ai modelli più grandi: ad un'analisi storica, anche sommaria, appare evidente come, a dispetto della loro esiguità numerica, siano state soprattutto le statue anatomiche "maggiori" a colpire i visitatori dei passati due secoli. Il manufatto che - vuoi per preziosità estetica, vuoi per valore scientifico-didattico, vuoi per provocatorietà - appare centrale rispetto alla collezione è la cosiddetta Venere de' Medici, in gran parte opera del ceroplasta Clemente Susini (1754-1814), che la realizzò sotto la guida dello scienziato Felice Fontana (1730-1805). La statua vuole essere una rivisitazione della omonima copia ellenistica da originale greco, conservata presso la Galleria degli Uffizi.

Qualiimodellidi riferimento? Le cerefemminilidella Specola, essendo sdraiate, fanno pensareallasculturafuneraria, valeadirealla decorazioneantropomorficadeisarcofaghi. La Venere in cera assomiglia alla figura giacente sulla Tomba della Beata Ludovica Albertoni, di Gian Lorenzo Bernini (1598-1680) (Kádár, Z., 1977, 525-31). Il suo corpo è infatti, come quello del precedente barocco, sdraiato e col capo leggermente reclinato, benché sia soprattutto nell'espressione del viso, tra agonia ed estasi, che le due statue rinvianoad unaispirazionecomune.Comelavergineberniniana, lanon-verginesusiniana sembra colta nell'atto di morire e, come lei, tradisce una sorta di malcelato orgasmo. La Venere sembra godere della propria sofferenza. Invita l'osservatore a rimirare le sue morbide carni e ad "entrare" in lei, asportando dal suo ventre un pezzo dopo l'altro. Le sue movenze, fintamente pudiche, eccitano l'amor profano. La collana di perle che le cinge il collo, la quale pure assolve la funzione di coprire il taglio sotto la gola, è una spia di lascivia. La Venere promana una sensualità contagiosa: è lo specimen che fornisce gli strumenti concettuali per leggere ogni corpo in chiave pan-erotica. La pittrice Elisabeth Vigée-Lebrun (1755-1842), che pure coi corpi nudi aveva una certa dimestichezza, accusò, nell'aprile 1792, un vero choc di fronte allo spettacolo che le venne presentato:

Questa vista mi fece un'impressione tale, che stavo per sentirmi male. Per molti giorni mi fu impossibile distrarmene, al punto che non potevo vedere una persona senza spogliarla mentalmente degli abiti e della pelle. Tutto cio mi mise in un odioso stato di nervosismo (Vigée-Lebrun, E., 1835-37, ii, 154).
La Venere dona all'osservatore il coraggio di gridare quanto, pur essendo sotto gli occhi di tutti, nessuno ardisce dichiarare: che cioè l'essere umano è nudo; che ogn soggetto può essere spogliato, persino della pelle; che è suscettibile di sventramento e penetrazione; chel'abitoèunameraconvenzioneborghese, incapacedicoprireunanudità ontologica. La Venere fa notare ciò che agli altri sfugge perché acuisce i sensi: "quando rividi Fontana, gli chiesi consiglio a che mi liberasse dall'importuna sensibilità dei miei organi: "sento troppo, gli dissi, vedo troppo e percepisco tutto anche da lontano,"”" commentò un'iperattiva Madame Vigée-Lebrun (Ibid.). Lo scompiglio dei sensi è dunque una sorta di rito di passaggio, che provoca la liberazione dell'immaginazione e, nel migliore dei casi, l'approdo ad una più solida maturità. Visitare una collezione di cere anatomiche è per una fanciulla una sorta di pre-deflorazione, da alcuni talvolta programmata. Denis Diderot (1713-84), che aveva preparato alle gioie del matrimonio sua figlia, facendole studiare le cere anatomiche della francese Marie Catherine Bihéron (1719-96), rifletteva: "sono quasi due mesi che è sposata; ha conservato e spero che conserverà per tutta la vita la semplicità, la dolcezza, la modestia di una fanciulla... $\mathrm{E}$ sapete a che deve questo privilegio...? A tre corsi di anatomia che ha fatto prima di passare nel letto nuziale da M.me Bihéron, tanto raccomandabile per il suo talento che per la sua saggezza" (Diderot, D. 1963, Lettera all'Abate Diderot, 13 Novembre1772, xii, 164). Le cere vanno oltre l'inganno del belletto femminile, rivelando quel nocciolo segreto della sessualità, il quale non dovrebbe mai uscire da una comunità rigorosamente monosessuale. "Si aprirono allora gli occhi di ambedue e conobbero che erano nudi; perciò cucirono delle foglie di fico e se ne fecero cinture," recita il Libro della Genesi. L'imbarazzo di fronte alla nudità nasce solo dopo aver conosciuto il peccato; ed è per questo che la altera Lady Marguerite Blessington (1789-1849) stimò queste "nudità e verità" così "spaventose e laide," da farle auspicare foglie di fico in chiave moderna, vale a dire l'istituzione di visite separate per uomini e donne:

Sono entrata oggi nel Gabinetto di Fisica e, benché vi sia rimasta solo qualche minuto, me ne sono andata con una sensazione di repulsione, che non mi ha più abbandonata. Sarebbe opportuno imporre restrizioni per impedire che questi modelli siano visti insieme da uomini e donne. [...] Non è opportuno che si abbiano sotto gli occhi i disgustosi dettagli dell'economia animale, in tutte le loro spaventose e laide nudità e verità (Blessington, M., 1839, 215).

Quale che sia la reazione alla vista della Venere - eccitazione, sdegno o svenimento -, dopo averla incontrata, non si rimane più gli stessi. Il cereo simulacro è infatti indecente e "asociale:" fa provare una vertigine, che è masochistica per le donne, sadica per gli uomini. Anche per questi ultimi, almeno a Firenze non così frequentemente medici, l'incontro con le cere femminili è una sorta di iniziazione sessuale, l'ingresso nel mondo degli adulti: è la catabasi in un "bordello anatomico," vale a dire in una sorta di teatro mnemotecnico di immagini fruibili a fini onanistici. 
I provvedimenti presi dalla direzione del museo, la quale ripose in teche di vetro chiuse a chiave questi oggetti che rischiavano di rovinarsi perché troppo frequentemente toccati dai visitatori, sembra confermarlo (Maerker, A., 2005, 81-96). La statua è scomponibile. Se è vero però che - come ammonisce il medico Lenardo negli Anni di peregrinazione di Wilhelm Meister di Johann Wolfgang Goethe (1749-1832) - "la costruzione è più istruttiva della distruzione, l'assemblare più che il separare," ciò vale, eventualmente, per il solo ceroplasta (Goethe, J. W., [1829] 1887-99, 64). Il visitatore partecipa infatti in maniera esclusiva ad una attività a questa speculare, cioè proprio a quella "distruzione" e a quel "separare" da cui si vuole rifuggire, il che non può che rinviare a suggestioni sadiche. Nelle Centoventi giornate di Sodoma, il Marchese De Sade (1740-1814) illustra così una delle sue più inquietanti perversioni:

Egli riceve la fanciulla in un gabinetto colmo di cadaveri di cera, molto ben imitati. Sono tutti dilaniati in modi differenti. Dice alla fanciulla di sceglierne uno, dal momento che lui la ucciderà ad immagine del cadavere le cui ferite a lei piacciono di più (Sade, A. F. D., [1782-85] 1986-91, i, 393).

La Venere è gravida; lo si scopre però solo alla fine della "visita." È l'ultima meraviglia che il peccaminoso manufatto offre allo spettatore: la donna, racchiusa in una teca di cristallo, è, come spesso avveniva nelle rappresentazioni anatomiche del corpo femminile, a sua volta teca o contenitore biologico. La verginità rimane in superficie, dunque. Impressionante è per l'osservatore moderno che in una cera, la quale, in seno ad una collezione anatomica, dovrebbe avere come scopo primario la rappresentazione naturalistica del corpo umano, si riscontri una assoluta incongruenza tra l'utero gravido e il resto del soggetto raffigurato. La Venere è a ben vedere, sotto il profilo morfologico, una donna non gravida nel cui utero è stato inserito un feto. La fanciulla non presenta infatti né il ventre dilatato né i seni appesantiti né alcuna delle alterazioni somatiche che accompagnano la gravidanza. Tali aspetti, che la avrebbero resa meno affascinante e avrebbero rovinato l'effetto sorpresa finale, vengono ignorati. Ad essere plasmata è pertanto una topografia ideale del corpo femminile, piuttosto che una verosimile rappresentazione degli spazi organici: l'indicazione di una potenzialità riproduttiva e non una reale gestazione. Anche il feto è, d'altro canto, troppo ben formato per essere così piccolo. La gravidanza è meramente simbolica: se ad essere ritratta è la donna nella pienezza fisiologica, questa non può essere incarnata che dallo stato gestante, anche a costo di imprecisioni anatomiche.

\section{UOMINI SCUOIATI}

Tra le figure maschili non ve n'è, a differenza di quanto accade per quelle femminili, una di particolare rilievo "emotivo." Notevole è però la cera impiegata per dar forma al sistema linfatico superficiale. In essa il protagonista cita l'Adamo della Cappella
Sistina; eppure, diversamente dal progenitore michelangiolesco, il quale tende l'indice verso il Creatore, egli ritrae il braccio, con un movimento che ricorda quello della statua dell'Aurora, sempre di Michelangelo, posta sulla tomba di Lorenzo de Medici, in Sacrestia Nuova. L'uomo sembra indicare se stesso e, con tal gesto, porsi al centro dell'universo o, più semplicemente, del "mondo anatomico." Da quest'ultimo è estromesso il Dio-Persona, ma, come si vedrà, non sarà espulsa la Divinità.

La raffigurazione di un "Adamo anatomico" era poco più che un topos della ceroplastica settecentesca e, in genere, dell'iconografia anatomica. L'artista-artigiano (o lo scienziato) si ergeva in tal modo al ruolo di demiurgo, di creatore dunque di una nuova specie ideal-tipica di uomini in cera. I modelli maschili a figura intera, non avendo né pelle, né capelli, né orpelli di sorta, permettono infatti, rispetto alle venerine, una più forte idealizzazione dei soggetti rappresentati, che diventano "tipi universali," al di fuori dello spazio e del tempo (Kleindienst, H., 1989, 225-32).

In che cosa si differenziano le statue maschili da quelle femminili? Nonostante fossero da tempo emerse diversità, soprattutto a livello osteologico, tra i corpi dei due sessi, le cere della Specola non sembrano tenerne conto, attribuendo, come di consueto, all'uomo il ruolo di soggetto anatomico unico. Non è pertanto seguendo questa strada che si possa procedere. È invece lecito individuare dicotomie intorno a snodi concettuali e rappresentativi, in grado di offrire un'immagine di quella cultura che produsse (e recepì) i modelli dei due sessi in cera (Jordanova, L., 1989, 43-86). Passionalità vs. apassionalità. Della dimensione sensuale e ammaliatrice del corpo femminile si è parlato. La donna è passionale: si lascia dominare dalle emozioni, che traspaiono dal volto. L'uomo, dal canto suo, nonostante la prestanza, non rivela una particolare carica erotica. Il suo sguardo è spento e spesso sbarrato. Se nelle rappresentazioni femminili a figura intera si cerca, con un movimento della gamba o del braccio, di nascondere le pudenda allo sguardo dei curiosi - la qual cosa le rende tanto più desiderabili -, nelle figure maschili esse sono mostrate con indifferenza. È come se l'uomo, già scuoiato, non avesse più nulla da perdere. Mentre la donna è "Venere", l'uomo è infatti "Marsia:" se la statua femminile eccita perché suggerisce una brutalizzazione che si può perpetrare in infinito, la statua maschile, quando in piedi, mostra i postumi di una violenza già consumata, quindi non ulteriormente prolungabile. L'apparato genitale dell'uomo è dunque un insieme di organi alla pari di qualunque altro e come tale è trattato. Il soggetto maschile è, come prevedeva il Neoclassicismo, atarassico, o, meglio, egli, pur provando dolore, si controlla per non esternare la sofferenza. Il corpo è teso come una corda di violino, ma lo sguardo non tradisce emozioni. Il contrasto lo rende sublime, come voleva l'estetica dell'epoca. Una differenziazione tra le statue maschili andrebbe però introdotta. Oltre a quelle in piedi, ve ne sono infatti di coricate. Queste ultime sembrano incarnare un modello 
di passionalità/passività, che è stato finora attribuito alle sole statue femminili. Le pose di tali uomini sono più "femminee:" un soggetto porta la mano dietro la nuca, quasi posasse per un ritratto da inviare ad un amante; un altro mostra il dorso in una inequivocabile allusione. In ciò alcuni interpreti hanno individuato componenti omoerotiche (Sheriff, M. D., 1996, 30-33). La contrapposizione dominante non sarebbe più tra uomo e donna, ma tra attivo/apassionale e passivo/passionale.

Visceralità vs. pre-superficialità. Anche sulla "visceralità" della rappresentazione femminile si è discusso. Le donne, secondo la medicina dell'epoca, erano particolarmente emotive, proprio perché animate da una fisiologia legata ai visceri. Le venerine si offrono sempre a qualcuno che tolga loro dalla cavità addominale intestini, polmoni e "frattaglie" varie. Il loro ventre è una sorta di grande piatto di portata, in cui l'ostensore affonda le mani per asportare organi dalla consistenza teoricamente gelatinosa. In questo guazzabuglio, l'utero è sicuramente il pezzo più importante: anche se non sempre gravido, occupa un posto centrale nella ideale topografia femminile. La donna è una scatola piena, dunque. L'uomo, di contro, è il modello d'elezione quando si desideri dar forma alla "pre-superficialità," vale a dire alla muscolatura più in vista (ma non alla pelle). Allorché scelto come soggetto per la resa di parti più interne, l'uomo è in genere "cavo," un contenitore senza contenuto. Ad esempio, nella statua che esibisce il sistema venoso, il modello pare svuotato. Al suo interno le uniche "frattaglie" rinvenibili sono il cuore e i reni: il soggetto maschile è infatti cardiocentrico, come quello femminile è isterocentrica. Tutto rimanda alla (pre)-superficie avvolgente, la quale è intessuta di vene: il didentro - una grande bolla d'aria che avvolge il cuore - esiste solo per enfatizzare il difuori. L'uomo è, in sintesi, una scatola vuota. Modularità vs. stratificazione. Le rappresentazioni del corpo seguono in genere due diversi schemi di visualizzazione: il topografico e il sistematico. Il primo rappresenta insieme tutti gli organi che si trovano in una data regione, come pure nervi, vasi e ciò che li connette a quanto è loro adiacente. La raffigurazione della donna, proprio in quanto scatola piena, segue tal tipo di prospettiva, di "varietà istologica." La statua femminile è modulare: la Venere è infatti composta di pezzi che si possono a poco a poco estrarre rimirare, studiare, rimettere a posto; le altre figure femminili, pur non scomponibili, danno la stessa impressione. L'uomo in cera ha una maggiore "unitarietà istologica" ed è per questo più spesso legato ad una rappresentazione sistematica, la quale tende a mettere insieme le parti facenti capo allo stesso sistema (solo ossa, muscoli, nerv ecc.), trascurando, per quanto possibile, il resto. Il corpo maschile non ha pezzi da portare via; è composto di apparati, che creano una stratificazione della sua superficie. Quando lo si vuole conoscere in profondità, lo si può solo esfoliare.

Morbidezza vs. durezza. La donna è scelta per dar forma a tessuti molli e cedevoli, in genere localizzati a livello addominale. Solo le cere femminili sono inoltre ricoperte di pelle, la quale offre un'illusione di morbida sensualità. L'uomo è invece scelto per esprimere tessuti duri: mai avvolto da pelle, il suo nucleo rappresentativo è costituito soprattutto dai muscoli. Vene e arterie hanno nel soggetto maschile un turgore e un'intensità cromatica, che le rendono talvolta poco credibili. Vasi linfatici e nervi sono a carico dell'uomo, forse perché effigiati come piccoli fili, i quali, come una pianta rampicante su un muro, si intrecciano a ridosso di una struttura rigida. I vasi linfatici scelgono tuttavia il corpo della donna quando si pongono nella cavità toracica e addominale: in questo caso offrono il destro per mostrare due scene di squartamento.

Orizzontalità vs. verticalità. La donna è sempre supina. Sdraiata, attende che il carnefice compia impietosamente la sua opera. Questo naturalmente non vuol dire che l'uomo in cera non conosca l'orizzontalità, ma che perlomeno ha la scelta tra le due opzioni. Gli organi maschili appaiono più solidi e per così dire capaci di reggersi da sé. Si mettano a confronto le due statue in cui vi è una rappresentazione "esplosa" dell'intestino. La prima, femminile, è la cosiddetta Sventrata: la donna, supina, mostra il proprio ventre dilaniato, da cui fuoriesce un materico intestino, che le collassa addosso. È, come sempre, ricoperta di pelle e il suo sguardo volge altrove, quasi che la fanciulla non volesse vedere la barbarie cui è stata sottoposta. La seconda, maschile, vede un uomo in piedi, il cui intestino, piuttosto smilzo, emerge dalla cavità addominale, restando ritto senza alcun sostegno. Il soggetto è senza pelle e il suo sguardo è quasi di sfida.

\section{RIFERIMENTI BIBLIOGRAFICI}

Sito della collezione di cere anatomiche del Museo della Specola di Firenze: http://www.specola.unifi.it/cere/

Aa. Vv, Encyclopaedia Anatomica, Taschen, Colonia-Londra-Madrid-New York-ParigiTokyo 1999

Aa. Vv, «La ceroplastica nella scienza e nell'arte», Atti del I Congresso Internazionale, Olschki, Firenze 1977, 2 voll.

Blessington M. contessa di, The Idler in Italy, Galignani, Parigi 1839.

Bucci M., Anatomia come arte, il Fiorino, Firenze 1976.

de Ceglia F. P., «Rotten corpses, a disembowelled woman, a flayed man. Images of the body from the end of the 17th to the beginning of the 19th century florentine wax models in the first-hand accounts of visitors», Perspectives on science, 2006.

Diderot D., Correspondance, ed. Roth G., Edition de minuit, Paris 1963.

Goethe J. W., Wilhelm Meisters Wanderjahre, ed. Neumann G., Dewitz H. G., in Sämtliche Werke, Deutscher Klassiker Verlag, Francoforte sul Meno, 1993, vol. X.

Hilloowala R. (a cura di), The Anatomical Waxes of La Specola, Arnaud, Firenze 1995 
Jordanova L., Sexual Visions. Images of Gender in Science and Medicine between the Eighteenth and Twentieth Centuries, The University of Wisconsin Press, Madison 1989

Kádár Z., "Sul profilo barocco della cosiddetta "Venere dei Medici" di cera», in Aa. Vv., La ceroplastica nella scienza e nell'arte. Atti del I Congresso Internazionale. Florence: Olschki, 1977, pp. 525-31

Kleindienst H., Ästhetisierte Anatomie aus Wachs. Ursprung, Genese, Integration, Tesi di dottorato, Univiversität Marburg, Marburgo 1989, 2 voll.

Knoefel P. K., «Florentine Anatomical Models in Wax and Wood», Medicina nei secoli, 1978 (15), pp. 329-40

Lanza B., Azzaroli Puccetti M. L., Poggesi M., Martelli A., Le Cere Anatomiche della Specola, Arnaud, Firenze 1997

Le Fur Y., Esthétique des cires anatomiques de Gaetano Giulio Zumbo (1656-1701) à Pierre Spitzner (1834-1896), Tesi di dottorato, Paris I Panthéon-Sorbonne, Parigi 1989

Lemire M., Artistes et mortels, Chabaud, Parigi 1990

Maerker A. «Uses and Publics of the Anatomical Model Collections of La Specola, Florence, and the Josephinum, Vienna, around 1800», in M. Beretta (ed.), From Private to Public. Natural Collections and Museums, Science History Publications, Usa 2005, pp. 81-96

Marei Krüger-Fürhoff I., «Der vervollständigte Torso und die verstümmelte Venus. Zur Rezeption antiker Plastik und plastischer Anatomie in Ästhetik und Reiseliteratur des 18. Jahrhunderts», Zeitschrift für Germanistik, n. f., 1998, (8), pp. 361-73.

Mazzolini R., «Plastic Anatomies and Artificial Dissections, in Models», The Third dimension of science, ed. de Chadarevian S., Hopwood N., Stanford University Press, Stanford 2004, pp. 43-70

Pyke E. J., A Biographical Dictionary of Wax Modellers, Clarendon Press, Oxford 1973-86, 4 voll

Sade A. F. D. marchese de, Les cent-vingt journées de Sodome, in Euvres complètes, J. J. Pauvert, Parigi 1986, vol. I.

Schnalke T., Disease in Wax. The History of Medical Moulage, Quintessence, Berlino 1995

Sheriff M. D., The Exceptional Woman. Elisabeth Vigée-Lebrun and the Cultural Politics of Art, University of Chicago Press, Chicago e Londra 1996.

Vigée Lebrun E., Souvenirs, H. Fournier, Parigi 1835-37, 3 voll. 